\title{
A SIMPLE PROOF OF A THEOREM OF CHACON
}

\author{
ROBERT CHEN
}

\begin{abstract}
A short and simple proof of a theorem of Chacon is presented by an application of a maximal inequality. A pointwise convergence theorem and the submartingale convergence theorem follow immediately from the theorem.
\end{abstract}

Here we present a short and simple proof of a theorem, due to Chacon [3], which implies a pointwise convergence theorem [1] and the submartingale convergence theorem [1], [2], [4].

THEOREM (CHACON). Let $\left\{X_{n}\right\}$ be a sequence of integrable random variables such that $\lim _{\inf _{n \rightarrow \infty}} E\left(\left|X_{n}\right|\right)<\infty$. Let

$$
X^{*}=\limsup _{n \rightarrow \infty} X_{n}, \quad X_{*}=\liminf _{n \rightarrow \infty} X_{n},
$$

and $T$ be the collection of all bounded stopping times. Then

$$
\underset{\tau, t \in T}{\lim \sup _{\tau}} E\left(X_{\tau}-X_{t}\right) \geqslant E\left(X^{*}-X_{*}\right)
$$

Further, if $\sup _{\tau \in T} E\left(\left|X_{\tau}\right|\right)<\infty$, then $X^{*}$ and $X_{*}$ are integrable.

Proof. By Lemma 1 of [1] and the Borel-Cantelli lemma, we can choose two strictly increasing sequences $\left\{\tau_{k}\right\}$ and $\left\{t_{k}\right\}$ of bounded stopping times such that $\lim _{k \rightarrow \infty} X_{\tau_{k}}=X^{*}$ almost surely and $\lim _{k \rightarrow \infty} X_{t_{k}}=X_{*}$ almost surely. Hence, the second assertion follows immediately from Fatou's lemma and we need only prove (1). To prove (1), it suffices to show that

$$
\sup _{\tau, t \in T} E\left(X_{\tau}-X_{t}\right) \geqslant E\left(X^{*}-X_{*}\right)
$$

It is also easy to see that, under the assumption of the theorem, if

Received by the editors February 10, 1976.

AMS (MOS) subject classifications (1970). Primary 60G40; Secondary 60F15, 60G99.

Key words and phrases. Bounded stopping time, Fatou's lemma, generalized sequence, Lebesgue's dominated convergence theorem, maximal inequality, submartingale convergence theorem. 
$\sup _{t \in T} E\left(\left|X_{t}\right|\right)=\infty$, then $\sup _{\tau, t \in T} E\left(X_{\tau}-X_{t}\right)=\infty$. Hence, we can, and do, assume that $\sup _{\tau \in T} E\left(\left|X_{t}\right|\right)<\infty$.

To prove (2), we need the following maximal inequality, which I learned from Chacon and Sucheston.

(3) $\lambda P\left(\left[\sup _{n}\left|X_{n}\right| \geqslant \lambda\right]\right) \leqslant \sup _{\tau \in T} E\left(\left|X_{\tau}\right|\right) \quad$ for each positive constant $\lambda$.

To see (3), let $M$ be a fixed positive integer and define a bounded stopping time $\tau$ by $\tau(w)=\inf \left\{n|1 \leqslant n \leqslant M,| X_{n}(w) \mid \geqslant \lambda\right\}, \tau(w)=M+1$ if no such $n$ exists, $w \in \Omega$. Then

$$
\lambda P\left(\left[\sup _{1 \leqslant n \leqslant M}\left|X_{n}\right| \geqslant \lambda\right]\right) \leqslant E\left(\left|X_{\tau}\right|\right) \leqslant \sup _{t \in T} E\left(\left|X_{t}\right|\right) .
$$

(3) follows immediately on letting $M \rightarrow \infty$.

Now let $\lambda$ be a positive constant, $\gamma(w)=\inf \left\{n|| X_{n}(w) \mid \geqslant \lambda\right\}, \gamma(w)=\infty$ if no such $n$ exists, $w \in \Omega$. Let $A=[\gamma<\infty], Y=\lambda \chi_{A^{c}}+\left|X_{\gamma} \chi_{A}\right|, Y_{n}=X_{n \wedge \gamma}$ for all $n \geqslant 1, Y^{*}=\lim \sup _{n \rightarrow \infty} Y_{n}$, and $Y_{*}=\lim _{\inf _{n \rightarrow \infty}} Y_{n}$. By Lemma 1 of [1] and the Borel-Cantelli lemma, we can choose two strictly increasing sequences $\left\{\tau_{k}\right\}$ and $\left\{t_{k}\right\}$ of bounded stopping times such that $\lim _{k \rightarrow \infty} Y_{\tau_{k}}=Y^{*}$ almost surely and $\lim _{k \rightarrow \infty} Y_{t_{k}}=Y_{*}$ almost surely. Since $\left|Y_{t}\right| \leqslant Y$ for all $t \in T$ and $E(Y) \leqslant \lambda+\sup _{t \in T} E\left(\left|X_{t}\right|\right)<\infty$, by Lebesgue's dominated convergence theorem, $\quad \lim _{k \rightarrow \infty} E\left(Y_{\tau_{k}}-Y_{t_{k}}\right)=E\left(Y^{*}-Y_{*}\right)$. So $\sup _{\tau, t \in T} E\left(Y_{\tau}-Y_{t}\right)$ $\geqslant E\left(Y^{*}-Y_{*}\right)$. Since $\left\{Y_{t} \mid t \in T\right\}=\left\{X_{t \wedge \gamma} \mid t \in T\right\}$ is a subset of $\left\{X_{t} \mid t \in T\right\}$, $\sup _{\tau, t \in T} E\left(X_{\tau}-X_{t}\right) \geqslant E\left(Y^{*}-Y_{*}\right)$. By (3), (2) follows on letting $\lambda \rightarrow \infty$ (since $X^{*}$ and $X_{*}$ are integrable).

Corollary 1 (Theorem 2 of [1]). Suppose that $E\left(\left|X_{n}\right|\right)<\infty$ for all $n \geqslant 1$ and $\lim _{\inf _{n \rightarrow \infty}} E\left(\left|X_{n}\right|\right)<\infty$. Consider the following two statements.

(a) The generalized sequence $\left\{E\left(X_{\tau}\right) \mid \tau \in T\right\}$ is convergent.

(b) $X_{n}$ converges almost surely to a finite limit.

Then (a) implies (b).

Corollary 2 (The submartingale Convergence theorem). Suppose that $\left\{X_{n}\right\}$ is a sequence of $L_{1}$-bounded random variables adapted to the increasing sequence $\left\{\mathscr{F}_{n}\right\}$ of $\sigma$-fields. Suppose that $E\left(X_{n+1} \mid \mathscr{F}_{n}\right) \geqslant X_{n}$ a.s. for all $n \geqslant 1$. Then $X_{n}$ converges almost surely to a finite limit.

REMARK. The theorem and Corollary 1 also hold under any one of the following two conditions.

(i) $\sup _{n} E\left(X_{n}^{+}\right)<\infty$.

(ii) $\sup _{n} E\left(X_{n}^{-}\right)<\infty$.

ACKNOWLeDgements. I would like to thank Professors Chacon and Sucheston for their valuable suggestions and comments. I also want to thank the referee for his invaluable and useful suggestions. 


\section{REFERENCES}

1. D. G. Austin, G. A. Edgar and A. Ionescu Tulcea (1974), Pointwise convergence in terms of expectations, Z. Wahrscheinlichkeitstheorie und Verw. Gebiete 30, 17-26. MR 50 \#11402.

2. J. R. Baxter (1974), Pointwise in terms of weak convergence, Proc. Amer. Math. Soc. 46, 395-398.

3. R. V. Chacon (1974), $A$ "stopped" proof of convergence, Advances in Math. 14, 365-368.

4. C. W. Lamb (1973), A short proof of the martingale convergence theorem, Proc. Amer. Math. Soc. 38, 215-217. MR 48 \#3119.

Department of Mathematics, University of Miami, Coral Gables, Florida 33124 